\title{
Vomiting, constipation, and abdominal pain during menstruation as first symptoms of Herlyn-Werner- Wunderlich syndrome: case report and literature review
}

\author{
Justyna Konys ${ }^{1,2}$, Jakub M. Kucharski ${ }^{1,2}$, Barbara Skowrońska², Aleksandra Banaszkiewicz², \\ Marcin Banasiuk ${ }^{2}$ \\ 'Students' Scientific Group GEKON, Department of Pediatric Gastroenterology and Nutrition, Medical University of Warsaw, Poland \\ 2Department of Pediatric Gastroenterology and Nutrition, Medical University of Warsaw, Poland
}

\begin{abstract}
Herlyn-Werner-Wunderlich syndrome, also known as OHVIRA (Obstructed Hemivagina and Ipsilateral Renal Anomaly), is an extremely rare abnormality of the Müllerian ducts, characterized by the presence of double uterus, unilateral cervico-vaginal obstruction and renal agenesis and/or other urinary tract anomalies. Most cases are diagnosed after menarche through an MRI, CT or ultrasound. There are no specific symptoms of this syndrome. Patients presented fever and vomiting during menstruation, intermenstrual bleeding and palpable abdominal, pelvic or vaginal mass. Thorough history taking is extremely important as common symptoms in pediatrics (vomiting, constipation, abdominal pain) may be a part of a more complex condition, not only within the GI tract but also including other systems.
\end{abstract}

KEY WORDS:

constipation, children, didelphys uterus, hematometrocolpos, renal agenesis.

\section{INTRODUCTION}

Obstructed hemivagina and ipsilateral renal anomaly (OHVIRA) syndrome (also known as Herlyn-Werner-Wunderlich syndrome) is a rare condition with very few cases reported. The abbreviation OHVIRA stands for obstructed hemivagina with ipsilateral renal agenesis. The third component of this disorder is didelphys uterus. The syndrome is the result of an impaired fusion of the Müllerian ducts. This fusion takes place during embryogenesis, between the 6th and 9th week after conception. The malformation is usually diagnosed after menarche [1].

Herlyn-Werner-Wunderlich syndrome is extremely rare. However, vomiting and constipation are relatively common in the pediatric population and usually indicate gastrointestinal (GI) tract disease. The case presented here was reported to emphasize that such widespread symptoms can be a part of a more complex condition that may occur within the GI tract, but also may include other systems. A short literature review was prepared to complement our report.

\section{CASE REPORT}

A 12-year-old girl was admitted to the Department of Pediatric Gastroenterology and Nutrition because of constipation (her last defecation was observed 2 days before admission with a very small amount of stool), dehydration, and abdominal pain. The patient reported pain in the left lower quadrant. It was still, non-radiating, and with no exacerbating factors. The pain began 2 days before admission.

On her first day in the hospital, the patient vomited (6 times) and refused to eat and drink because of the pain.

\section{ADDRESS FOR CORRESPONDENCE:}

Justyna Konys, Department of Pediatric Gastroenterology and Nutrition, Medical University of Warsaw,

Poland, e-mail: justyna.konys@gmail.com 


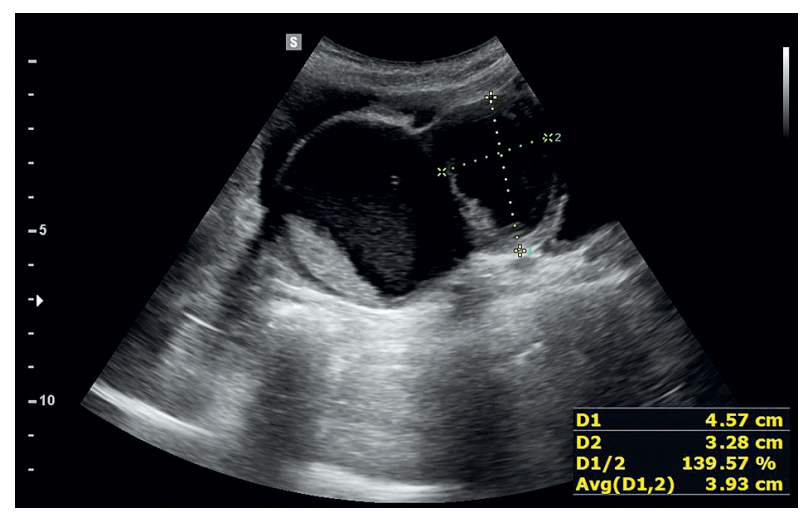

FIGURE 1. Ultrasonography of cystic lesions localized in the lesser pelvis

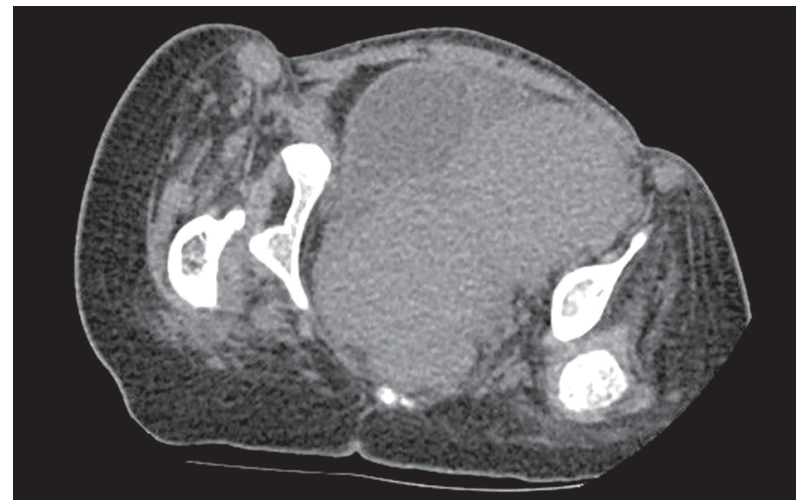

FIGURE 2. Contrast CT presenting a double-bodied uterus with hematometrocolpos on the left side

The patient was experiencing menstruation (which started 2 days before admission and was the third menstruation cycle in the patient's life). A gradual reduction in menstrual bleeding was observed. Physical examination revealed a palpable mass in the left iliac fossa. This was suspected to be a mass of stool. The patient was previously hospitalized because of myelomeningocele and hydrocephalus. She has a ventriculoperitoneal shunt, scoliosis, kyphosis, left kidney agenesis, and neurogenic bladder dysfunction. She is also underweight (below 1 percentile for her age group based on the growth chart).

The ultrasonography performed at the Emergency Department revealed numerous cystic lesions localized in the lesser pelvis, some of which were filled with hyperechogenic content (Figure 1). It also showed the presence of dense content in the uterine cavity and some fluid in the peritoneal cavity. Evaluation of the abdomen was difficult because of the peculiar anatomical conditions, thus CT scan was scheduled. Contrast CT was performed and showed a double-bodied uterus. The right side of the uterus had no pathologies, while hematometrocolpos was present in the left side of the uterus (Figure 2). CT scan also revealed fluid in the peritoneal cavity, ventriculoperitoneal drainage, ovarian cysts in the left ovary (the biggest measuring $4 \mathrm{~cm}$ ), agenesis of the left kidney, and multiple skeletal deformations.
An enema was used to relieve constipation (for 3 days), laxatives were changed from lactulose to macrogols, and the patient was discharged home. A visit to the Gynecological Outpatient facility was scheduled. Gynecological consultation resulted in the initiation of continuous oral contraceptives (microgynon). Surgical treatment was also scheduled. Surgical treatment of the syndrome usually consists of opening the blind hemivagina and performing fluid drainage. Two weeks after the gynecological consultation (but still prior to surgery), the patient returned to the Department of Pediatric Gastroenterology and Nutrition with acute abdominal pain in the same area as previously observed. She was transported to the Department of Gynecology for urgent surgery.

\section{DISCUSSION}

The first case of a patient with OHVIRA syndrome was described in 1950 [3]. A more in-depth description of this disease was conceived more than 20 years later by Herlyn and Werner, and subsequently expanded upon by Wunderlich in $1976[4,5]$. On this basis, the syndrome was given its second name, Herlyn-Werner-Wunderlich syndrome.

The etiology of OHVIRA syndrome is not clear. However, it is hypothesized to be the result of the failed fusion of the Müllerian ducts during embryogenesis (8th week). These ducts develop to form female reproductive organs. The newest theory claims that the maldevelopment of the Müllerian ducts can also result in the absence of the kidney and the ureter $[1,2]$. There hasn't been found any genetic mutation or karyotype abnormality predisposing to the development of the syndrome.

The incidence of congenital uterine anomalies is estimated to be $4.3-6.7 \%$. OHVIRA accounts for $0.1-3.8 \%$ of congenital uterine anomalies and is the most rare type $[2,6]$. In the vast majority of cases, OHVIRA syndrome is diagnosed in young, adolescent girls after the first menstrual bleeding [7, 8]. An analysis of 27 cases revealed that the median age at diagnosis was 14 years old [9]. It has been previously reported that this syndrome can also be diagnosed during pregnancy, birth process $[2,10,11,12]$, or incidentally during diagnostic imaging for other reasons [13, 14].

Obstructed hemivagina and ipsilateral renal anomaly syndrome, beyond the typical presentation as intermenstrual bleeding, abdominal pain, pelvic or abdominal mass, and renal agenesis, can include symptoms from multiple organs [1]. Additional symptoms described in the literature include acute abdomen symptoms [15], ectopic ureter $[8,16,17]$, pyocolpos $[2,18]$, skeletal anomaly (ectrodactyly [19], kyphoscoliosis [20]) and wandering spleen [21]. In our case, constipation and vomiting were an unusual first presentation. 
Ultrasonography is the predominant first-choice imaging method. It is very helpful in the detection of hematocolpos, but this method cannot identify the type of Müllerian duct anomaly [22]. The advantages of ultrasonography include availability, cost, and the absence of radiation. However, ultrasonography has a high operator dependency and such rare malformations as OHVIRA syndrome may go undiagnosed. The best diagnostic choice for ultrasound access is transvaginal. Given the fact that in most cases the symptoms occur in childhood and early puberty, transvaginal ultrasonography cannot be performed before the beginning of sexual activity.

Magnetic resonance imaging (MRI) is the gold standard for diagnosing Müllerian duct anomaly (MDA) and allows for the identification of the specific type of MDA [22]. MRI is most useful in cases where differentiation of the uterine anomaly is not easily achievable by ultrasonography or physical exam. This method is characterized by higher sensitivity in the detection of the uterine morphology and the continuity of the vagina when compared to ultrasonography [15]. Moreover, MRI is the best method to identify associated pathologies (endometriosis, pelvic adhesions, other viscera anomalies) [22].

Laparoscopy should be considered when MRI imaging is not available or ultrasonography and MRI diagnosis is not clear. Indications for laparoscopy include: interval between menarche and diagnosis, severity of the symptoms, presence of hematometra or pyometra [22].

An additional advantage of laparoscopy is that it can be used for therapeutic purposes, such as drainage of hematocolpos/hematometrocolpos, septectomy, or marsupialization of the blind hemivagina [22].

The treatment of choice for OHVIRA syndrome is single-stage vaginoplasty. In rare cases, such as extreme proximal vaginal septum or infectious complications, hemihysterectomy may be an alternative [23]. In recent years, there has been a trend towards publications on hymen-sparing surgeries. Sleiman describes safe and successful laparoscopy-assisted resection for the management of high vaginal septum while maintaining an intact hymen [24]. Another hymen-sparing management was demonstrated recently, namely the transrectal ultrasound (TRUS)-guided vaginoscopic septoplasty supported by pre- and postoperative diagnostics with the use of a novel ultrasound technique: three-dimensional saline-solution infusion contrast sonovaginocervicography (3D-SVC) with virtual speculoscopy [25].

Due to the development of diagnostics and treatment methods, patients can manage to avoid complications such as infertility. Ugurlucan et al. reported the follow-up after surgical treatments in which 7 cases out of 32 (21.9\%) had married and 5 of these $(71.4 \%)$ were pregnant or had delivered [23]. After hysteroscopy, patients achieve a close to normal prognosis for the outcome of their pregnancies $(75 \%$ term delivery rates and $85 \%$ live birth rates) [26]. Successful pregnancy outcomes in OHVIRA syndrome are estimated at 57\%-68\% [2].

In our case in differential diagnosis must be consider infectious diseases of the digestive tract, functional constipation, irritable bowel syndrome, gynecological tumors, Mayer-Rokitansky-Kuster-Hauser syndrome (MRKH) or another MDA.

The present case shows that differential diagnosis is not always obvious and that a common list of manifestations can also indicate a rare disease. Thanks to solid and accurate history taking, unique disorders may be overlooked less frequently.

\section{DISCLOSURE}

The authors declare no conflict of interest.

\section{REFERENCES}

1. Albulescu DM, Ceauşescu AE, Sas LM, et al. The Herlyn-Werner-Wunderlich triad (OHVIRA syndrome) with good pregnancy outcome - two cases and literature review. Rom J Morphol Embryol 2018; 59: 1253-1262.

2. Tangshewinsirikul C, Dulyaphat W, Diawtipsukon S, et al. Successful pregnancy outcome in Herlyn-Werner-Wunderlich syndrome with pyocolpos: A case report and literature review. Clin Case Rep 2020; 8: 2217-2222.

3. Embrey MP. A Case of Uterus Didelphys with Unilateral Gynatresia. Br Med J 1950; 1: 820-821.

4. Herlyn U, Werner H. Simultaneous occurrence of an open Gartner-duct cyst, a homolateral aplasia of the kidney and a double uterus as a typical syndrome of abnormalities. Geburtshilfe Frauenheilkd 1971; 31: 340-347.

5. Wunderlich M. Unusual form of genital malformation with aplasia of the right kidney. Zentralbl Gynakol 1976; 98: 559-562.

6. Saravelos SH, Cocksedge KA, Li TC. Prevalence and diagnosis of congenital uterine anomalies in women with reproductive failure: a critical appraisal. Hum Reprod Update 2008; 14: 415-429.

7. Kapczuk K, Friebe Z, Iwaniec K, et al. Obstructive Müllerian anomalies in menstruating adolescent girls: a report of 22 cases. J Pediatr Adolesc Gynecol 2018; 31: 252-257.

8. Han TI, Kim I-O, Kim WS, et al. US identification of the anal sphincter complex and levator ani muscle in neonates: infracoccygeal approach. Radiology 2000; 217: 392-394.

9. Smith NA, Laufer MR. Obstructed hemivagina and ipsilateral renal anomaly (OHVIRA) syndrome: management and follow-up. Fertil Steril 2007; 87: 918-922.

10. Agarwal A, Agarwal S, Sharma A. OHVIRA syndrome in post-cesarean period: An exclusive clinical scenario managed by twostaged operative procedure. Taiwan J Obstet Gynecol 2018; 57: 613-615.

11. Muraoka A, Tsuda H, Kotani T, et al. Severe hemoperitoneum during pregnancy with obstructed hemivagina and ipsilateral renal anomaly syndrome: a case report. J Reprod Med 2016; 61: 290-294.

12. Dural O, Tas IS, Ugurlucan FG, et al. Unique Diagnosis of Obstructed Hemivagina and Ipsilateral Renal Anomaly (OHVIRA) at the Time of First-Trimester Ultrasound Imaging. J Pediatr Adolesc Gynecol 2020; 33: 748-751.

13. Panwar VK, Narain TA, Mittal A, et al. Female counterpart of the Zinner syndrome in men: decoding the "OHVIRA" syndrome. BMJ Case Rep 2021; 14. 
13. Demir MK, Savas Y, Furuncuoglu Y, et al. Imaging Findings of the Unusual Presentations, Associations and Clinical Mimics of Acute Appendicitis. Eurasian J Med 2017; 49: 198-203.

15. Gungor Ugurlucan F, Bastu E, Gulsen G, et al. OHVIRA syndrome presenting with acute abdomen: a case report and review of the literature. Clin Imaging 2014; 38: 357-359.

16. Balık AÖ, Yağcı B, Özoğul M, et al. A case report of first hearing loss, then painful menarche: a young girl with Herlyn-Werner-Wunderlich syndrome (OHVIRA syndrome) and concomitant inner ear anomalies. J Obstet Gynaecol 2021; 1-3.

17. Yuan P, Qi L, Wang L. Incontinence after vaginal septum resection for a missed diagnosis of ectopic dysplastic kidney and ureter. Int Urogynecol J 2017; 28: 645-646.

18. Sleiman Z, Zreik T, Bitar R, et al. Uncommon presentations of an uncommon entity: OHVIRA syndrome with hematosalpinx and pyocolpos. Facts Views Vis Obgyn 2017; 9: 167-170.

19. Cappello S, Piccolo E, Cucinelli F, et al. Successful preterm pregnancy in a rare variation of Herlyn-Werner-Wunderlich syndrome: a case report. BMC Pregnancy Childbirth 2018; 18: 498.

20. Jhirwal M, Singh P, Sharma C, et al. Herlyn-Werner-Wunderlich (HWW) syndrome with kyphoscoliosis: a rare urogenital anomaly in a teenage girl. BMJ Case Rep 2021; 14.

21. Thomas AK, Thomas R. The likely association between wandering spleen and absent left kidney. Emerg Radiol 2021; 28: 431-435.

22. Del Vescovo R, Battisti S, Di Paola V, et al. Herlyn-Werner-Wunderlich syndrome: MRI findings, radiological guide (two cases and literature review), and differential diagnosis. BMC Med Imaging 2012; $12: 4$.

23. Gungor Ugurlucan F, Dural O, Yasa C, et al. Diagnosis, management, and outcome of obstructed hemivagina and ipsilateral renal agenesis (OHVIRA syndrome): Is there a correlation between MRI findings and outcome? Clin Imaging 2020; 59: 172-178.

24. Sleiman Z, Wehbe GS, Rassy EE, et al. A Novel Surgical Intervention for an Uncommon Entity: Laparoscopy-Assisted Resection of a Vaginal Septum in Obstructed Hemivagina and Ipsilateral Renal Anomaly Syndrome. J Laparoendosc Adv Surg Tech A 2019; 29: 714-716.

25. Ludwin A, Ludwin I, Bhagavath B, et al. Virginity-sparing management of blind hemivagina in obstructed hemivagina and ipsilateral renal anomaly syndrome. Fertil Steril 2018; 110: 976-978.

26. Grimbizis GF, Camus M, Tarlatzis BC, et al. Clinical implications of uterine malformations and hysteroscopic treatment results. Hum Reprod Update 2001; 7: 161-174. 